\title{
Nasopharyngeal carcinoma: A new synthesis of literature data (Review)
}

\author{
DANIELA JICMAN (STAN) $)^{1,2^{*}}$, ELENA NICULET ${ }^{3-5}$, MIHAELA LUNGU $^{6,7^{*}}$, CRISTIAN ONISOR $^{3}$, \\ LAURA REBEGEA $^{7}$, DOINITA VESA ${ }^{1,8}$, LAURA BEZMAN ${ }^{3,9}$, FLORIN CIPRIAN BUJOREANU ${ }^{5,10}$, \\ MIHAELA IONELA SARBU ${ }^{11}$, RAUL MIHAILOV ${ }^{8}$, SILVIA FOTEA $^{6,12^{*}}$ and ALIN LAURENTIU TATU ${ }^{5,6,9,13}$
}

${ }^{1}$ Department of Otorhinolaryngology, 'Sfantul Apostol Andrei’ Emergency Clinical Hospital, 800578 Galati;

${ }^{2}$ Biomedical Doctoral School and ${ }^{3}$ Department of Morphological and Functional Sciences, Faculty of Medicine and Pharmacy,

'Dunarea de Jos' University of Galați, 800010 Galati; ${ }^{4}$ Department of Pathology, 'Sfantul Apostol Andrei' Emergency Clinical

Hospital, 800578 Galati; ${ }^{5}$ Multidisciplinary Integrated Center of Dermatological Interface Research MIC-DIR,

'Dunarea de Jos' University, 800010 Galati; ${ }^{6}$ Department of Neurology, 'Sfantul Apostol Andrei' Emergency Clinical Hospital, 800578 Galati; ${ }^{7}$ Clinical Medical Department and ${ }^{8}$ Clinical Surgical Department, Faculty of Medicine and Pharmacy,

'Dunarea de Jos' University, 800010 Galati; ${ }^{9}$ Department of Ophthalmology, 'Sfantul Apostol Andrei' Emergency

Clinical Hospital, 800578 Galati; ${ }^{10}$ Department of Dermatology, 'Sfanta Cuvioasa Parascheva' Clinical Hospital of

Infectious Diseases, 800179 Galati; ${ }^{11}$ Faculty of Medicine and Pharmacy, 'Dunarea de Jos' University, 800010 Galati;

${ }^{12}$ Department of Pediatrics, 'Sf. Ioan' Clinical Hospital for Children, 800487 Galati; ${ }^{13}$ Research Center in the Field of

Medical and Pharmaceutical Sciences, ReFORM, 'Dunarea de Jos' University of Galati, 800010 Galati, Romania

Received August 6, 2021; Accepted September 6, 2021

DOI: $10.3892 / \mathrm{etm} .2021 .11059$

Abstract. Nasopharyngeal carcinoma (NPC) is an epithelial tumor, which develops most frequently from the lateral

Correspondence to: Dr Elena Niculet, Department of Morphological and Functional Sciences, Faculty of Medicine and Pharmacy, 'Dunărea de Jos' University of Galați, 35 Alexandru Ioan Cuza Street, 800010 Galati, Romania

E-mail: helena_badiu@yahoo.com

Dr Florin Ciprian Bujoreanu, Department of Dermatology, 'Sfanta Cuvioasa Parascheva' Clinical Hospital of Infectious Diseases, 393 Traian Street, 800179 Galati, Romania

E-mail: florin.bujoreanu@gmail.com

${ }^{*}$ Contributed equally

Abbreviations: 2D-RT, two-dimensional radiotherapy; AJCC, American Joint Committee on Cancer; anti-IL-6R mAb, anti-human IL-6R monoclonal antibody; DNA, deoxyribonucleic acid; EBER, EBV-encoding region; EBV, Epstein-Barr virus; HIV, human immunodeficiency virus; HNC, head and neck cancers; HPV, human papilloma virus; HT, helical tomotherapy; IL-6, interleukin-6; IMRT, intensity-modulated radiation therapy; LMP1, latent membrane protein 1; MMP-2, matrix metalloproteinase 2; MMP-9, matrix metalloproteinase 9; NPC, nasopharyngeal carcinoma; ORL, otorhinolaryngology; WHO, World Health Organization

Key words: nasopharyngeal carcinoma, Epstein Barr virus, histopathology, radiotherapy, chemotherapy, surgery, genetic modification pharyngeal recess and holds some complex epidemiological characteristics. Its unusual race and geographic distribution suggests that not only the environmental factors are a contributing factor to the development of this rare cancer type, but also the genetic traits play an important role, along with nitrosamine-containing food consumption and Epstein-Barr virus infection. The signs and symptoms which a patient can present and suffer from are various and include nasal, otic, neurological as well as general ones; the way this tumor manifests being dependent on the stage of the tumor. The therapeutic management applicable in NPC needs to be established according to the case of the patient and include radiotherapy, chemotherapy, surgery, immune therapy, targeted therapy or combined treatment. The main objective of the treatment is local and regional tumor control; relapse is an important factor for future development of distant metastases. New therapeutic concepts are always sought of, current research focusing on precision medicine, meaning systemic treatment with a personalized radiotherapy approach according to the characteristics of the tumor.

\section{Contents}

1. Introduction

2. Materials and methods

3. Epidemiology and etiology

4. Histogenesis

5. Signs and symptoms

6. Classification, staging and diagnosis

7. Genetic components 
8. Relation to other associated pathologies and medication

9. Management

10. Conclusions

\section{Introduction}

At present, epidemiological data supports the fact that head and neck tumors are a major public health problem due to their continuously rising incidence, prevalence and death rate. Head and neck cancers (HNC) account for over 550,000 cases and 380,000 cancer-related deaths worldwide, annually (1). Most otorhinolaryngology (ORL) tumors have an insidious, asymptomatic development, thus being either an incidental finding, or a late diagnosis of advanced, therapeutically unmanageable tumors $(2,3)$.

The region of the body usually known as 'head and neck' includes the superior aero-digestive tract, which incorporates the oral cavity, paranasal sinuses, pharynx, larynx, cervical esophagus, thyroid, associated lymph nodes, soft tissues and bones. The various tissues from this anatomical region give rise to a large spectrum of tumors and tumor-like conditions (4). One of the most unusual malignant tumors among $\mathrm{HNC}$ is the nasopharyngeal carcinoma (NPC).

The nasopharynx is part of the upper airway system and an anatomical element connecting the nasal cavities with the larynx and trachea, through the oropharynx. It is the most superior part of the pharynx, having as a superior anatomical limit the skull base and as an inferior one, the soft palate. It comprises important local and regional anatomic elements such as the pharyngeal orifice of the Eustachian tube, Rosenmüller fossae and Gerlach's tubal amygdala. The most frequent malignant tumor found at this level is NPC, an epithelial tumor which develops from Rosenmüller's fossa (5).

\section{Materials and methods}

The present review is the result of a thorough literature research which included studies from 1979 to 2021, using databases such as Web of Science and Google Scholar with the help of keywords such as 'nasopharyngeal carcinoma' alone or associated with others key words such as 'treatment' or 'etiology'. Other articles and means of information were selected, after they had been deemed useful for our research, such as the World Wide Web, using sites as WebMD or NCBI. In total, 58 studies were included in the present review.

\section{Epidemiology and etiology}

A distinctive entity among HNC, NPC is characterized by a distinct worldwide distribution (6), being considered endemic in the southern region of China where the incidence is $\sim 15-50 / 100,000$ inhabitants, and also in Indonesia, Malaysia and South-East Asia (7).

Causal factors for NPC development include: tobacco, alcohol and nitrosamine-containing food consumption. Alcohol, especially when it is combined with tobacco, has an increasing effect as regards the risk of developing this type of cancer. The aforementioned factors have a multiplying, synergistic effect on the risk for oral cavity, pharyngeal and laryngeal cancer development $(8,9)$. Similarly, other incriminating factors in the development of these cancer types are considered to be a rich diet in salt preserved fish and meat (9), host genetics (HLA gene on the 6p21 chromosome), environmental factors (such as chemical substances or carcinogens) as well as the Epstein-Barr virus (EBV) or human papilloma virus (HPV; the high-risk subtypes: 16, 18, 31, 33 and 35) infection. Extremely rare cases have been also linked to the human immunodeficiency virus (HIV) infection; one case presented the patient as being co-infected with EBV. Much as the Koebner phenomenon in skin disorders, NPC develops in a prior set environment, with certain characteristics which support malignant growth (5,10-15).

A first step in NPC development is considered to be the stable infection with EBV in the nasopharyngeal epithelium. Similarly, at the epithelial cell level, EBV infection manifests through lithic replication; in NPC development, slow replication appears to be the dominant infective manner (16). EBV slowly infects epithelial cells at this level, and sporadically enters the productive lithic viral infection. In this manner, type II latency is maintained, and EBV gene expression is limited to mRNA which is coded by these oncogenes: BNA1, LMP1, LMP2, EBER and BART (17). The expression of lithic EBV genes has been detected along with the latent membrane protein 1 (LMP1) which can induce genomic instability and stimulate immune-suppressive cytokine release in order to evade immune recognition of the viral infected cells. As such, latent genes and lithic ones are involved in the malignant transformation of the nasopharyngeal epithelium (16).

One of the most studied oncoproteins is LMP1; it is considered to be the main EBV oncoprotein, which on the one hand can induce important genetic and epigenetic alterations, and on the other hand can promote cellular mobility, invasion and cervical lymph node metastasis, a unique characteristic of NPC $(18,19)$.

In short, the oncogenic activity of EBV is revealed by the important effects exerted on the host genome, modifying its genetic profile, inducing genomic instability, evading host immunity and promoting cell survival through LMP1 activity. Another important fact stems from the activity of EBV; being able to induce stem-cell like properties in NPC tumor cells (16).

Inflammation is a key player in the cytokine release phenomena, and also in tumor development, progression and migration. Literature data suggests that malignant cells use chemokine gradients in order to metastasize to other body sites. Interleukin-6 (IL-6) appears to have a role in NPC progression, as it has the capacity of promoting malignant behavior. It is involved in NPC and also other types of malignant tumors: pancreatic, breast, prostate and lung cancers; it influences the capacity of invasiveness and migration of NPC cells by increasing it through mediation of matrix metalloproteinases 2 and 9 (MMP-2 and MMP-9). IL-6 and its receptors are highly expressed in NPC cells (20-23). Chow et al revealed that NPC patients have higher IL-6 levels as compared with the average, healthy individuals, and have stated that NPC tumor cells may be also a source for IL-6 (23). Targeting its receptor could be at least an associated treatment in NPC; the monoclonal, anti-human IL-6R antibody (anti-IL-6R mAb) having inhibitory effects on the malignant cell proliferation, and on the migrating and invasive capacities of NPC cells (20-23). 


\section{Histogenesis}

NPC develops from the surface of crypt epithelium found in the mucous layer of the nasopharynx. In some cases, this tumor can stem from the basal layers of the stratified squamous epithelium, a fact supported still by the strong immune reactivity for $\mathrm{p} 63$, both in the tumor and the normal basal cells (16).

\section{Signs and symptoms}

The signs and symptoms that NPC patients can present with are according to the stage and extension of the tumor, and can be grouped as follows:

The nasal level: unilateral nasal obstruction, epistaxis, nasal voice, olfactory acuity decrease, and olfactory disorders which can range even as severe as cacosmia. Almost $80 \%$ of patients suffer from nasal signs and symptoms $(7,24)$.

The otic level: recurrent middle ear infections with ototubal dysfunction, hypoacusis to cophosis, ear fullness sensation, otorrhea, otorrhagia, middle ear effusion and tinnitus $(7,24)$.

Neurological signs and symptoms develop in accordance to the tumor extension and implicitly, to the nerve structures which it involves: vestibule-cochlear nerve, facial and abducens nerve, with certain sensibility loss $(7,24)$.

Lymph node enlargement can also accompany the evolution of NPC, localizing at the cervical or submandibular level, or even at distant sites $(7,24)$.

General signs and symptoms which the NPC patient can develop are: headaches, low fever, anemia and weight loss $(7,24)$.

In the early stages of NPC development the signs and symptoms are nonspecific, setting in slowly and the most frequent ones are otic, nasal or ocular. The following time frame is characterized by an increase in the intensity and persistence of symptoms, this especially taking place as the tumor expands and as it extends intracranially.

Having in mind the characteristics presented earlier, NPC appears to have a propensity for distant metastasis, with a higher frequency than other types of HNC. In the order of their frequency, metastases can be localized in the bones, liver, lungs and distant lymph nodes (25).

\section{Classification, staging and diagnosis}

The World Health Organization (WHO) classifies NPC by its histopathological characteristics in the following types: keratinizing type (up to 25\%), non-keratinizing differentiated type (almost $15 \%$ ) and non-keratinizing undifferentiated (up to 65\%) (7).

EBV-associated, undifferentiated NPC has certain special characteristics such as: invasiveness, distant metastases, and sensitivity to both radiotherapy and chemotherapy. Differentiated NPC, which is not associated with EBV, is similar to other ORL cancers, having a mostly local and regional development (a distant one to a lesser extent), and is less sensitive to chemo- and radiotherapy (17).

NPC staging is conducted according to the eighth edition of the American Joint Committee on Cancer (AJCC) staging $(26,27)$.
The primary tumor can range from an in situ one (Tis), to an extensive infiltrating one (with intracranial, orbital, hypopharyngeal, parotid or soft tissue involvement) (T4). Lymph node involvement $(\mathrm{N})$ can range from absent regional lymph node metastases (NO), to uni-/bilateral cervical lymph node metastases (over $6 \mathrm{~cm}$ ) and/lymph node metastases extending under the cricoid cartilage (N3). Distant metastases can be absent (cM0), present (cM1) or present and with microscopic confirmation (pM1). The anatomic stage and prognostic groups stem from the earlier characterization of the tumor and its behavior, ranging from stage 0 to stage IVC (7).

It has already been established that the terms pM0 and $\mathrm{Mx}$ are not taken into consideration for tumor staging; these are frequently used in the pathologic (p) or clinical (c) classification, which are useful in those cases where surgical intervention is the most suitable therapeutic option (26).

In order to diagnose this type of cancer which is mostly a late finding, it is necessary to perform an elaborate local examination with the help of a posterior rhinoscopy or endoscopy, associated with a biopsy obtained from key-tissue changes. Imaging techniques can help in rhinopharyngeal tumor diagnosis by accurately describing the tumor diameter, its precise location and whether there is extension to the neighboring structures (5). As a plus, EBV detection can be a useful marker for diagnosis (28).

The most simple and reliable method of diagnosing the presence of EBV is by using the in situ hybridization for the EBV-encoding region (EBER), which is present in abundance in the latent-infected EBV cells. Less reliable is the use of the polymerase chain reaction in order to search for EBV in the tumor cells, due to the fact that even low numbers of EBV-positive lymphocytes can generate a false-positive result (29).

The latest medical research has accentuated the need for plasma EBV deoxyribonucleic acid (DNA) liquid biopsy in population screening (30).

\section{Genetic components}

Genetic factors in NPC development are considered co-factors, along with EBV infection and food consumption containing nitrosamines. Some genes that are considered to play a role in the development of this type of cancer are: HLA class I genes, TP53 and MDM2 (cell cycle control genes), MMP2 (cell adhesion/migration gene) and RAD51L1 (DNA repair gene). There is a great deal of research required in finding a genetic background for NPC, although the specialty literature has consistent findings with regard to the aforementioned genes (31).

\section{Relation to other associated pathologies and medication}

Statins are an already well-known class of drugs having the capacity to induce pro-apoptotic and anti-proliferative activities in cancer cells, being thus able to act as anticancer drugs. In NPC, the molecular mechanism and the possible effects of statins are yet unknown, although research conducted by Ma et al (2019) has revealed promising results (32). Simvastatin, an inactive prodrug before being hydroxylated in the gastrointestinal system, used as a chemotherapy treatment agent, had 
positive effects on an NPC cell line by reducing the viability of the cell line (in a dose-dependent manner), increasing enzymatic activity (caspase-3), and also inducing apoptosis through cell-arresting effects in the G1 cycle phase $(32,33)$.

Chronic ORL diseases have been associated with an increased risk for NPC. Patients who used nasal drops or even aspirin were revealed to have a two-fold risk of developing this type of cancer. These chronic diseases, along with the related drugs had no significant effect concerning NPC development if more than 5 years has passed since their occurrence. Important factors appear to be the untreated, chronically evolving nasal polyps, the uncured, chronic ORL diseases, the younger patients at first diagnosis and the recent or even first-time aspirin use. Another medication which is positively involved in cancer development is hydrochlorothiazide, a diuretic or saluretic which is associated with squamous cell carcinoma located on the lip $(34,35)$.

Beta-blockers, mainly used for heart failure, hypertension, intention tremors, but also for dermatologic diseases including hemangiomas, Kaposi sarcoma, melanoma, or pemphigus are generally considered safe. Their activities are exerted by blocking the $\beta$-adrenergic receptors in the body, where they are abundant in numerous organs or tissues. Some research has revealed that these receptors are also expressed by malignant cells or immune cells, and by activating them, consequent changes could take place with regard to the tumor microenvironment. This indicates that they influence tumor proliferation, migration and also invasion. That, being said, some studies revealed that beta-blocker use did not in fact influence the incidence of head and neck cancer, while another study reported that propranolol reduced the viability of head and neck cancer, inducing apoptosis and decreasing production of VEGF (36-39). Further studies need to be conducted in order to find the precise implications and consequences that beta-blockers have on NPC.

Tetracycline, a drug which also possesses anti-inflammatory properties, with TNF- $\alpha$ inhibition, is used either alone or in combination with other drugs and it has the capacity to decrease the synthesis of energy in a cell, and thus, block cell division. It therefore has a potential therapeutic ability in cancer cases, possibly used as a carrier for various medications towards the cancerous cells or even by coupling them with radionuclides for use in local radiotherapy. Tetracycline appears to have cytostatic effects in NPC or laryngeal cancers and a study conducted by Leezenberg et al in 1979, revealed that patients suffering from these types of cancers, when administered tetracycline in association with other therapies, had a longer survival $(40,41)$.

Concerning diseases that are associated with NPC, studies have revealed that connective tissue diseases, such as scleroderma or dermatomyositis, are found in patients suffering from such a malignancy. Connective tissue diseases, similarly to diabetes, are a burden for the organism in terms of prevalence and impairment. The mechanism by which NPC and connective tissue diseases are linked remains unknown, but there are hypotheses that state that one may influence the development of the other; dermatomyositis being occasionally diagnosed as paraneoplastic. Furthermore, another hypothesis is the possible immune dysregulation with abnormal recognition of tissues. The fact that malignant tumors develop from normal cells of the body that suffer various genetic alterations, may be the link between NPC development and autoimmune diseases such as connective tissue ones. This could also explain the association between two or more autoimmune diseases (such as the association between thyroiditis, vitiligo and alopecia aerata or multiple autoimmune syndrome type 3). Diabetes is known to act both as a risk and prognostic factor in malignancy development; it is a disease which favors infection and inflammation, with important medical and social consequences. In NPC, Peng et al revealed that diabetes mellitus patients had a poorer prognosis, with lower disease-free survival rates, more frequent loco-regional relapse and lower overall survival rates (42-45).

\section{Management}

The therapeutic approach of NPC should be personalized for each patient, according to some factors such as the time of diagnosis (early or late diagnosis), tumor stage, tumor grade and histopathology type, depth of tumor invasion, performance status, local expertise availability, the general health state of patients, associated pathology, and preferences.

The therapeutic possibilities that can be applied according to the case of the patient, are: radiotherapy, chemotherapy, surgery, immune therapy, targeted therapy or combined treatment. The main objective of the treatment is a favorable local and regional control of the tumor, as it was found that relapse is an important factor in the development of distant metastases (18).

Most NPC are radiosensitive, this being an important treatment factor. Although conventional two-dimensional radiotherapy (2D-RT) can successfully control T1-T2 tumors in $75-90 \%$ of cases, and T3-T4 in 50-75\% of cases (without distant metastases), there is a great deal of inconvenience.

Due to its positioning, the nasopharynx is surrounded by a series of anatomical elements which are extremely radiosensitive including the medulla oblongata, spinal cord, eyes, middle and internal ear, parotid gland, temporal lobes and the hypothalamus-pituitary axis. The main characteristic of NPC is the fact that it infiltrates rapidly in the neighboring structures, and thus the radiation volumes are irregular, with multiple toxicities to the elements involved by the tumor (18).

In the latest years, techniques have evolved, going from conventional $2 \mathrm{D}$ or $3 \mathrm{D}$, to intensity-modulated radiation therapy (IMRT). IMRT is an advanced form of RT which modulates radiation in order to closely target the tumor; it adapts the radiation dose according to the shape of the tumor, its center receiving the highest dose of radiation and decreasing its intensity towards the periphery. It allows a local tumor control in $90 \%$ of patients, being the new standard therapy for NPC. That being said, helical tomotherapy (HT) is another innovative type of RT, that similar to IMRT, is based on inverse planning, but it uses a rotational gantry system and not fixed numbers of beam angles. Chen et al reported important dosimetric outcomes with improved patient outcomes with the use of HT, but further studies need to be performed in this direction $(18,46)$.

Another innovative RT treatment is brachytherapy, which uses removable implants that are placed in the tumor area and that are extracted after the adequate dose has been 
administered. Frequent radioactive substances used are iridium 192, cesium, radium or cobalt. Some studies which researched the dose response found that higher radiation doses determine improved local tumor control, but also increase the rate of severe complications (palate and/or sphenoid floor perforation and nasopharynx necrosis). Brachytherapy has favorable outcomes for primary and recurrent NPC, it is well tolerated and has minimal morbidity (47-49).

Radiotherapy is also used when other therapies have failed, or when the tumor has relapsed (7). A recent concept is adaptive radiotherapy which uses one or more sessions with the purpose of correcting some variables that are patient-derived and that can develop during treatment, this in order to optimize the dose distribution in every patient. The beneficial roles of this new concept are toxicity decrease and local control improvement in advanced carcinomas, however time is required in order for it to be introduced as routine practice (50).

NPC was determined to also be sensitive to chemotherapy, and radiotherapy sensitivity is correlated highly with chemotherapy sensitivity. The standard of care in locally and regionally advanced cases of NPC is concomitant platinum-based chemotherapy $(7,18,51)$. That being said, induction chemotherapy appears to be superior to concomitant chemotherapy alone (30). Administered before chemotherapy treatment, concomitant radiotherapy has the benefit of maintaining a good local, regional and distant control, results comparable to IMRT (52).

Over time, various treatment combinations have been studied, however, cisplatin is at the basis of NPC treatment, in doses of $100 \mathrm{mg} / \mathrm{m}^{2}$ every 3 weeks (7). The main manner by which platinum-based chemotherapy works is by damaging the cancer cell DNA through the inducement of apoptosis. Although it is considered to be the treatment basis, cisplatin can have important toxicities including ototoxicity, nephrotoxicity, nausea, vomiting, liver enzyme increases, peripheral neuropathy, or bone marrow inhibition; if it is administered concurrently with radiotherapy, it can exacerbate acute toxicities such as mucositis or dermatitis (53).

New research studies are awaited with regard to the efficacy of gemcitabine administered in association with cisplatin as induction chemotherapy treatment in local and regional advanced NPC (52).

Other chemotherapeutic agents that can be used are: cetuximab, 5-fluorouracil or docetaxel. Monoclonal antibodies that are newly approved are: nivolumab and pembrolizumab (53). As it is already known, immune therapy uses agents that have the capacity to block immune control check-points; it is considered as a revolution in treating advanced metastatic and recurrent cancers. The current research focuses on the efficacy of ipilimumab which mainly targets CTLA-4 and is used in combination with nivolumab in the treatment of rare tumors which also include NPC (30).

In treating NPC, surgical intervention is considered to be a safe option (7). As an anatomical region which is difficult to approach surgically, the nasopharynx is rarely a surgically approached region; it is located close to some vital vascular and nerve structures such as foramen ovale, foramen lacerum, foramen spinosum, foramen jugulare and the carotid canal. That being said, when necessary and recommended, the nasopharynx can be surgically treated through a trans-palatine-maxillary-cervical approach, a trans-mandibular approach, a transcervical-mandibular-palatine approach, through the infratemporal fossa, a lateral temporal approach (although with a significant failure rate of 15.6-58\%), or an endoscopic approach $(7,54)$.

The main treatment approach of NPC is radiotherapy, more importantly, IMRT, which is frequently administered in association with chemotherapy; for recurrent tumors, brachytherapy is the main treatment method, with the risk of skull base radiotherapy necrosis development $(47,55)$. Stereotactic radiosurgery can also be used (24). Although radical dissection of cervical lymph nodes is indicated in recurrent or persistent NPC cases, numerous patients only receive palliative chemotherapy (18).

In addition to the aforementioned IL-6R blockers, recent research has focused on melatonin, a compound with oncostatic properties which modulates the angiogenic, proliferative and metastatic properties of some cancers, including the HNC. Stanciu et al (2020) found that patients with oral squamous cell carcinoma had decreased levels of melatonin and that this hormone can be used in the assessment of cancer progression, being a predictive biomarker for metastatic spread; they also stipulated that melatonin could be used as a possible therapeutic agent for preventing tumor progression (56).

NPC treatment is multidisciplinary. Irrespective of the selected therapeutic approach, the main treatment purpose is tumor eradication with minimization of adverse effects, the improvement of the quality of life of the patient and relapse prevention. At present, new concepts are introduced with regard to anticancer treatment, including the use of precision medicine, which refers to administering a systemic treatment and personalized radiotherapy according to tumor characteristics (57).

\section{Conclusions}

NPC is an epithelial tumor with one of the most aggressive behaviors among HNC, having high rates of invasion and metastasis and frequent failure of treatment. It is a radiosensitive malignant tumor and radiotherapy remains the basis for treatment; combined treatments, also using chemotherapy, have yielded improved disease-free survival rates. With more accurate radiotherapy techniques, local and regional tumor control has been improved, while new treatment options are necessary for metastatic spread management (such as IL-6R blockers).

\section{Acknowledgements}

Not applicable.

\section{Funding}

The present work was supported by the 'Dunarea de Jos' University of Galati, Romania, through the research center, Multidisciplinary Integrated Center of Dermatological Interface Research MIC-DIR [Centrul Integrat Multidisciplinar de Cercetare de Interfata Dermatologica (CIM-CID)] and the internal grant (grant no. RF3668/01.10.2021). 


\section{Availability of data and materials}

The information generated and analyzed during the current study is available from the corresponding author on reasonable request.

\section{Authors' contributions}

DJS, EN, ALT, ML, SF, FB, CO and LR contributed to the acquisition, analysis and interpretation of data. DJS, EN, ALT, ML, SF, FB, DV and LB made substantial contributions to the conception and design of the work, while DJS, EN, ALT, ML, SF, FB, MIS and RM supervised and substantially revised this work. DJS and EN confirm the authenticity of all the raw data. All authors have read and approve the published version of the manuscript.

\section{Ethics approval and consent to participate}

Not applicable.

\section{Patient consent for publication}

Not applicable.

\section{Competing interests}

The authors declare they have no competing interests.

\section{References}

1. Khetan P, Boffetta P, Luce D, Stucker I, Curado MP, Menezes A, Wunsch-Filho V, Ahrens W, Lagiou P, Serraino D, et al: Occupations and the risk of head and neck cancer: A pooled analysis of the international head and neck cancer epidemiology (INHANCE) consortium. J Occup Environ Med 61: 397-404, 2019.

2. Moro JDS, Maroneze MC, Ardenghi TM, Barin LM and Danesi CC: Oral and oropharyngeal cancer: Epidemiology and survival analysis. Einstein (Sao Paulo) 16: eAO4248, 2018 (In English, Portuguese).

3. Hashim D and Boffetta P: Head and neck cancers. In: Occupational Cancers. Anttila S and Boffetta P (eds). Springer, Cham, Switzerland, pp57-105, 2020.

4. Cramer JD, Burtness B, Le QT and Ferris RL: The changing therapeutic landscape of head and neck cancer. Nat Rev Clin Oncol 16: 669-683, 2019.

5. Mankowski NL and Bordoni B: Anatomy, head and neck, nasopharynx. [Updated 2021 Feb 7]. In: StatPearls [Internet]. Treasure Island (FL): StatPearls Publishing; Available from: https://www ncbi.nlm.nih.gov/books/NBK557635. Accessed March 13, 2021.

6. Chen YP, Chan ATC, Le QT, Blanchard P, Sun Y and Ma J: Nasopharyngeal carcinoma. Lancet 394: 64-80, 2019.

7. Shah AB, Zulfiqar $\mathrm{H}$ and Nagalli S: Nasopharyngeal carcinoma. [Updated 2020 Sep 8]. In: StatPearls [Internet] Treasure Island (FL): StatPearls Publishing. Available from: https://www.ncbi.nlm.nih.gov/books/NBK554588. Accessed March 13, 2021

8. Niaz K, Maqbool F, Khan F, Bahadar H, Ismail Hassan F and Abdollahi M: Smokeless tobacco (paan and gutkha) consumption, prevalence, and contribution to oral cancer. Epidemiol Health 39: e2017009, 2017.

9. Pichardo G: Nasopharyngeal cancer. [20.01.2020]. In: WebMD.com [Internet]. Available from: https://www.webmd com/cancer/nasopharyngeal-cancer. Accessed March 13, 2020.

10. Boda D, Docea AO, Calina D, Ilie MA, Caruntu C, Zurac S, Neagu M, Constantin C, Branisteanu DE, Voiculescu V, et al: Human papilloma virus: Apprehending the link with carcinogenesis and unveiling new research avenues (Review). Int J Oncol 52: 637-655, 2018.
11. Nwabudike LC and Tatu AL: Reply to Happle R et al. Koebner's sheep in Wolf's clothing: Does the isotopic response exists as a distinct phenomenon? J Eur Acad Dermatol Venereol 32: e336-e337, 2018

12. Xing B, Qiao XF, Qiu YH and Li X: TMPO-AS1 regulates the aggressiveness-associated traits of nasopharyngeal carcinoma cells through sponging miR-320a. Cancer Manag Res 13: 415-425, 2021.

13. Manfredi R, Sabbatani S, Gianelli U and Marinacci G: Epstein-Barr virus associated nasopharyngeal carcinoma and local polymorphic B-cell lymphoproliferative disorder in a patient with HIV disease. J Int Assoc Physicians AIDS Care (Chic) 6: 255-259, 2007

14. Hald $\mathbf{J}$ and Larsen PL: Nasopharyngeal carcinoma in an HIV-positive patient causing severe morbidity and early death. J Laryngol Otol 107: 149-150, 1993.

15. Draganescu M, Baroiu L, Iancu A, Dumitru C, Radaschin D, Polea ED, Bobeica C, Tatu AL, Niculet E and Fekete GL: Perspectives on skin disorder diagnosis among people living with HIV in southeastern Romania. Exp Ther Med 21: 97, 2021.

16. Tsao SW, Tsang CM and Lo KW: Epstein-Barr virus infection and nasopharyngeal carcinoma. Philos Trans R Soc Lond B Biol Sci 372: 20160270, 2017.

17. Nakanishi Y, Wakisaka N, Kondo S, Endo K, Sugimoto H, Hatano M, Ueno T, Ishikawa K and Yoshizaki T: Progression of understanding for the role of Epstein-Barr virus and management of nasopharyngeal carcinoma. Cancer Metastasis Rev 36: 435-447, 2017.

18. Kieser A and Sterz KR: The latent membrane protein 1 (LMP1) Curr Top Microbiol Immunol 391: 119-149, 2015.

19. Nwabudike LC and Tatu AL: Reply to Gambichler T et al: Altered epigenetic pathways and cell cycle dysregulation in healthy appearing skin of patients with koebnerized squamous cell carcinomas following skin surgery. J Eur Acad Dermatol Venereol 33: e3-e4, 2019.

20. Sun W, Liu DB, Li WW, Zhang LL, Long GX, Wang JF, Mei Q and $\mathrm{Hu}$ GQ: Interleukin-6 promotes the migration and invasion of nasopharyngeal carcinoma cell lines and upregulates the expression of MMP-2 and MMP-9. Int J Oncol 44: 1551-1560, 2014.

21. Niculet E, Chioncel V, Elisei AM, Miulescu M, Buzia OD, Nwabudike LC, Craescu M, Draganescu M, Bujoreanu F, Marinescu E, et al: Multifactorial expression of IL-6 with update on COVID-19 and the therapeutic strategies of its blockade (Review). Exp Ther Med 21: 263, 2021.

22. Solomon I, Voiculescu VM, Caruntu C, Lupu M, Popa A, Ilie MA, Albulescu R, Caruntu A, Tanase C, Constantin C, et al: Neuroendocrine factors and head and neck squamous cell carcinoma: An affair to remember. Dis Markers 2018: 9787831, 2018.

23. Chow KC, Chiou SH, Ho SP, Tsai MH, Chen CL, Wang LS and Chi KH: The elevated serum interleukin- 6 correlates with the increased serum butyrate level in patients with nasopharyngeal carcinoma. Oncol Rep 10: 813-819, 2003.

24. Low JS, Chua ET, Gao F and Wee JT: Stereotactic radiosurgery plus intracavitary irradiation in the salvage of nasopharyngeal carcinoma. Head Neck 28: 321-329, 2006.

25. Liao W, Tian M and Chen N: Characteristic and novel therapeutic strategies of nasopharyngeal carcinoma with synchronous metastasis. Cancer Manag Res 11: 8431-8442, 2019.

26. Deng F and Gaillard F: Nasopharyngeal cancer (staging). Available at: https://radiopaedia.org/articles/nasopharyngeal-cancer-staging?lang=us. Accessed March 14, 2020.

27. Guo R, Mao YP, Tang LL, Chen L, Sun Y and Ma J: The evolution of nasopharyngeal carcinoma staging. Br J Radiol 92: 20190244, 2019.

28. Song $C$ and Yang S: A meta-analysis on the EBV DNA and VCA-IgA in diagnosis of nasopharyngeal carcinoma. Pak J Med Sci 29: 885-890, 2013

29. Tsai ST, Jin YT, Mann RB and Ambinder RF: Epstein-Barr virus detection in nasopharyngeal tissues of patients with suspected nasopharyngeal carcinoma. Cancer 82: 1449-1453, 1998.

30. Lam WKJ and Chan JYK: Recent advances in the management of nasopharyngeal carcinoma. F1000Res 7 (F1000 Faculty Rev): 1829,2018

31. Hildesheim A and Wang CP: Genetic predisposition factors and nasopharyngeal carcinoma risk: A review of epidemiological association studies, 2000-2011: Rosetta stone for NPC: Genetics, viral infection, and other environmental factors. Semin Cancer Biol 22: 107-116, 2012.

32. Ma Z, Wang W, Zhang Y, Yao M, Ying L and Zhu L: Inhibitory effect of simvastatin in nasopharyngeal carcinoma cells. Exp Ther Med 17: 4477-4484, 2019. 
33. Nwabudike LC, Elisei AM, Buzia OD, Miulescu M and Tatu AL: Statins. A review on structural perspectives, adverse reactions and relations with non-melanoma skin cancer. Rev Chim (Bucharest) 69: 2557-2562, 2018.

34. Xiao X, Zhang Z, Chang ET, Liu Z, Liu Q, Cai Y, Chen G, Huang QH, Xie SH, Cao SM, et al: Medical history, medication use, and risk of nasopharyngeal carcinoma. Am J Epidemiol 187: $2117-2125,2018$

35. Pottegård A, Hallas J, Olesen M, Svendsen MT, Habel LA, Friedman GD and Friis S: Hydrochlorothiazide use is strongly associated with risk of lip cancer. J Intern Med 282: 322-331, 2017.

36. Kim MS, Han KD and Kwon SY: Pre-diagnostic beta-blocker use and head- and neck cancer risk: A nationwide population-based case-control study. Medicine (Baltimore) 98: e16047, 2019.

37. Tatu AL,Elisei AM, Chioncel V, Miulescu M and Nwabudike LC: Immunologic adverse reactions of $\beta$-blockers and the skin. Exp Ther Med 18: 955-959, 2019.

38. Wolter NE, Wolter JK, Enepekides DJ and Irwin MS: Propranolol as a novel adjunctive treatment for head and neck squamous cell carcinoma. J Otolaryngol Head Neck Surg 41: 334-344, 2012.

39. Zhang L, Mai HM, Zheng J, Zheng JW, Wang YA, Qin ZP and Li KL: Propranolol inhibits angiogenesis via down-regulating the expression of vascular endothelial growth factor in hemangioma derived stem cell. Int J Clin Exp Pathol 7: 48-55, 2013.

40. Leezenberg JA, Wesseling H and Kroon AM: Possible cytostatic action of tetracyclines in the treatment of tumors of the nasopharynx and larynx. Eur J Clin Pharmacol 16: 237-241, 1979.

41. Nwabudike LC and Tatu AL: Response to -chronic exposure to tetracyclines and subsequent diagnosis for non-melanoma skin cancer in a large Mid-Western US population. J Eur Acad Dermatol Venereol 32: e159, 2018.

42. Ferris RL and Koch WM: Connective tissue disease coincident with nasopharyngeal carcinoma: Two sporadic cases in a Western population. Arch Otolaryngol Head Neck Surg 129: 101-105, 2003.

43. Tatu AL and Ionescu MA: Multiple autoimmune syndrome type 3-thyroiditis, vitiligo and alopecia areata. Acta Endocrinol (Buchar) 13: 124-125, 2017.

44. Earar K, Sirbu I, Onisor C and Luca E: Oral rehabilitation on implants and introduction of pathogenic mechanisms in relation to oral implants-sugar diabetes. Rev Chim 70: 3750-3752, 2019.

45. Peng XS, Xie GF, Qiu WZ, Tian YH, Zhang WJ and Cao KJ: Type 2 diabetic mellitus is a risk factor for nasopharyngeal carcinoma: A 1:2 matched case-control study. PLoS One 11: e0165131, 2016

46. Chen AM, Yang CC, Marsano J, Liu T and Purdy JA: Intensity-modulated radiotherapy for nasopharyngeal carcinoma: Improvement of the therapeutic ratio with helical tomotherapy vs segmental multileaf collimator-based techniques. Br J Radiol 85: e537-e543, 2012.
47. Lee N, Hoffman R, Phillips TL, Xia P, Quivey JM, Weinberg V and Hsu IC: Managing nasopharyngeal carcinoma with intracavitary brachytherapy: One institution's 45-year experience. Brachytherapy 1: 74-82, 2002.

48. Teo P, Leung S, Lee W and Zee B: Intracavitary brachytherapy significantly enhances local control of early T-stage nasopharyngeal carcinoma: The existence of a dose-tumor-control relationship above conventional tumoricidal dose. Int J Radiat Oncol Biol Phys 46: 445-458, 2000.

49. Cheah SK, Lau FN, Yusof MM and Phua VC: Treatment outcome with brachytherapy for recurrent nasopharyngeal carcinoma. Asian Pac J Cancer Prev 14: 6513-6518, 2014.

50. Castelli J, Simon A, Lafond C, Perichon N, Rigaud B, Chajon E, De Bari B, Ozsahin M, Bourhis J and de Crevoisier R: Adaptive radiotherapy for head and neck cancer. Acta Oncol 57: 1284-1292, 2018.

51. Zhang Y, Sun Y and Ma J: Induction gemcitabine and cisplatin in locoregionally advanced nasopharyngeal carcinoma. Cancer Commun (Lond) 25: 39, 2019.

52. Lee VH, Lam KO, Chang AT, Lam TC, Chiang CL, So TH, Choi CW and Lee AW: Management of nasopharyngeal carcinoma: Is adjuvant therapy needed? J Oncol Pract 14: 594-602, 2018.

53. Sindhu SK and Bauman JE: Current concepts in chemotherapy for head and neck cancer. Oral Maxillofac Surg Clin North Am 31: 145-154, 2019.

54. Tsang RK and Wei WI: Salvage surgery for nasopharyngeal cancer. World J Otorhinolaryngol Head Neck Surg 1: 34-43, 2015.

55. Kam MK, Teo PM, Chau RM, Cheung KY, Choi PH, Kwan WH, Leung SF, Zee B and Chan AT: Treatment of nasopharyngeal carcinoma with intensity-modulated radiotherapy: The Hong Kong experience. Int J Radiat Oncol Biol Phys 60: 1440-1450, 2004.

56. Stanciu AE, Zamfir-Chiru-Anton A, Stanciu MM, Stoian AP, Jinga V, Nitipir C, Bucur A, Pituru TS, Arsene AL, Dragoi CM, et al: Clinical significance of serum melatonin in predicting the severity of oral squamous cell carcinoma. Oncol Lett 19: 1537-1543, 2020

57. Kaidar-Person O, Gil Z and Billan S: Precision medicine in head and neck cancer. Drug Resist Updat 40: 13-16, 2018.

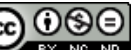

This work is licensed under a Creative Commons Attribution-NonCommercial-NoDerivatives 4.0 International (CC BY-NC-ND 4.0) License. 